\title{
Formulation of a Mathematical Model for Transmission and Control of Zika Virus Fever Dynamics
}

\section{${ }^{* 1}$ ADEYEMO, KA; ${ }^{2}$ AKINWANDE, NI; ${ }^{3}$ ABDULRAHMAN, S; ${ }^{4}$ KUTA, FA}

\author{
${ }^{I}$ Department of Computer Science and Mathematics, Nigeria Police Academy, Wudil, Nigeria. \\ ${ }^{2}$ Department of Mathematics, Federal University of Technology, Minna, Nigeria. \\ ${ }^{3}$ Department of Mathematics, Federal University, Birni Kebbi, Nigeria. \\ ${ }^{4}$ Department of Microbiology, Federal University of Technology, Minna, Nigeria. \\ Corresponding Author Email: k.2a.adeyemo@gmail.com, +2348035161740
}

\begin{abstract}
We formulated a mathematical model for the transmission and control of zika virus fever dynamics, incorporating controls; and mosquito bite, sexual contact, vertical transmission blood transfusion as medium of transmissions. Based on our previous findings that the model is mathematically well posed for analysis, the state variables at the endemic state are expressed in terms of parameters and forces of infection, substitution and simplifying led to a polynomial. Invoking Descartes sign rule of polynomial confirmed the existence of a unique endemic equilibrium point of the model if and only if the effective reproduction number is greater than one $\left(R_{e}>1\right)$.
\end{abstract}

DOI: https://dx.doi.org/10.4314/jasem.v22i10.15

Copyright: Copyright $(92018$ Adeyemo et al. This is an open access article distributed under the Creative Commons Attribution License (CCL), which permits unrestricted use, distribution, and reproduction in any medium, provided the original work is properly cited.

Dates: Received: 10 September 2018; Revised: 12 October 2018; Accepted: 29 October 2018

Keywords: Zika virus fever; Control; Medium of transmission; Unique endemic equilibrium point

Zika virus derives its name from zika forest in Uganda where it was first discovered in monkey in 1947 , in a female mosquito in 1948 and in human in 1952; the infection caused by zika virus is called zika virus fever which does not kill but is linked with Microcephaly and Neurological anomalies (WHO, 2016). Zika virus lives longer in the semen than in the blood or virginal fluid (CDC, 2016). The virus is transmitted through mosquito bite, sexual contact, vertical transmission and blood transfusion (ECDC, 2016). (Funk et al., 2016) worked on comparative analysis of Zika and Dengue by setting the viruses. (Kucharski et al, 2016) worked on the outbreaks of zika in French Polynesia between 2013 and 2014. Gao et al., (2016) and Augusto et al., (2017) modeled zika virus as mosquito borne and sexually transmitted disease. The aim of this paper is to establish the condition for the existence of the endemic state and also determine the number of the endemic points of our improved model equations.

\section{MATERIALS AND METHODS}

Total female population $\left(N_{1}\right)$ is split into: Susceptible female compartment $\left(S_{1}\right)$, Exposed female compartment $\left(E_{1}\right), \quad$ Symptomatic female compartment $\left(I_{11}\right), \quad$ Asymptomatic female compartment $\left(I_{12}\right)$ and the Removed female compartment $\left(R_{1}\right)$. The male population $\left(N_{2}\right)$ is similarly partitioned into sub -populations as given in equation (15); and mosquito population is split into mosquitoes without zika virus $\left(S_{3}\right)$ and mosquitoes with zika virus $\left(I_{3}\right)$ as in (14).

\section{MODEL EQUATIONS}

$$
\begin{aligned}
& \frac{d S_{1}}{d t}=\theta_{1} \omega_{1} \Lambda_{1}-\frac{S_{1}}{N_{1}}\left\{\alpha_{1} \phi_{3} I_{3}+\alpha_{21} \phi_{21} I_{21}\left(1-\epsilon_{c} \tau_{c}\right)+\alpha_{22} \phi_{22} I_{22}\left(1-\epsilon_{c} \tau_{c}\right)\right\}-\mu_{1} S_{1} \\
& \frac{d E_{1}}{d t}=\left(1-\theta_{1}\right) \omega_{1} \Lambda_{1}+\frac{S_{1}}{N_{1}}\left\{\alpha_{1} \phi_{3} I_{3}+\alpha_{21} \phi_{21} I_{21}\left(1-\epsilon_{c} \tau_{c}\right)+\alpha_{22} \phi_{22} I_{22}\left(1-\epsilon_{c} \tau_{c}\right)\right\}-\left(\gamma_{1}+\sigma_{11}+\sigma_{12}+\mu_{1}\right) E_{1} \\
& \frac{d I_{11}}{d t}=\sigma_{11} E_{1}-\left(\gamma_{11}+\mu_{1}\right) I_{11}
\end{aligned}
$$


$\frac{d I_{12}}{d t}=\sigma_{12} E_{1}-\left(\gamma_{12}+\mu_{1}\right) I_{12}$
$\frac{d R_{1}}{d t}=\left(1-\omega_{1}\right) \Lambda_{1}+\gamma_{1} E_{1}+\gamma_{11} I_{11}+\gamma_{12} I_{12}-\mu_{1} R_{1}$

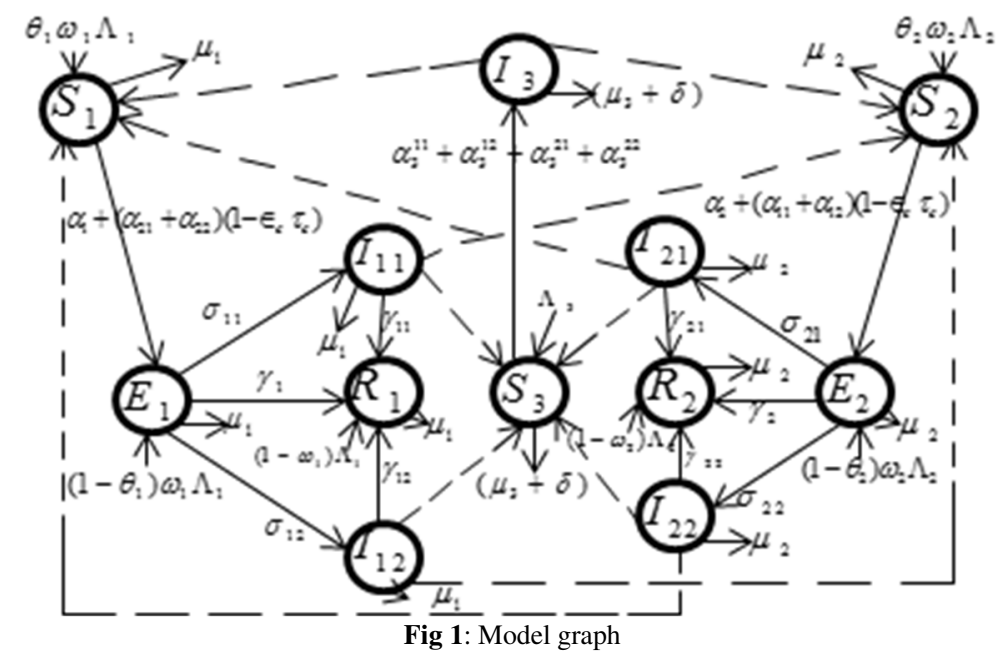

$$
\begin{aligned}
\frac{d S_{3}}{d t} & =\Lambda_{3}-\frac{S_{3}}{N_{3}}\left(\alpha_{3}^{11} \phi_{11} I_{11}+\alpha_{3}^{12} \phi_{12} I_{12}+\alpha_{3}^{21} \phi_{21} I_{21}+\alpha_{3}^{22} \phi_{22} I_{22}\right)-\left(\mu_{3}+\delta\right) S_{3} \\
\frac{d I_{3}}{d t} & =\frac{S_{3}}{N_{3}}\left(\alpha_{3}^{11} \phi_{11} I_{11}+\alpha_{3}^{12} \phi_{12} I_{12}+\alpha_{3}^{21} \phi_{21} I_{21}+\alpha_{3}^{22} \phi_{22} I_{22}\right)-\left(\mu_{3}+\delta\right) S_{3} \\
\frac{d S_{2}}{d t} & =\theta_{2} \omega_{2} \Lambda_{2}-\frac{S_{2}}{N_{2}}\left\{\alpha_{2} \phi_{3} I_{3}+\alpha_{11} \phi_{11} I_{11}\left(1-\epsilon_{c} \tau_{c}\right)+\alpha_{12} \phi_{12} I_{12}\left(1-\epsilon_{c} \tau_{c}\right)\right\}-\mu_{2} S_{2} \\
\frac{d E_{2}}{d t} & =\left(1-\theta_{2}\right) \omega_{2} \Lambda_{2}+\frac{S_{2}}{N_{2}}\left\{\alpha_{2} \phi_{3} I_{3}+\alpha_{11} \phi_{11} I_{11}\left(1-\epsilon_{c} \tau_{c}\right)+\alpha_{12} \phi_{12} I_{12}\left(1-\epsilon_{c} \tau_{c}\right)\right\}-\left(\gamma_{2}+\sigma_{21}+\sigma_{22}+\mu_{2}\right) E_{2} \\
\frac{d I_{21}}{d t} & =\sigma_{21} E_{2}-\left(\gamma_{21}+\mu_{2}\right) I_{21} \\
\frac{d I_{22}}{d t} & =\sigma_{22} E_{2}-\left(\gamma_{22}+\mu_{2}\right) I_{22} \\
\frac{d R_{2}}{d t} & =\left(1-\omega_{2}\right) \Lambda_{2}+\gamma_{2} E_{2}+\gamma_{21} I_{21}+\gamma_{22} I_{22}-\mu_{2} R_{2} \\
N_{1} & =S_{1}+E_{1}+I_{11}+I_{12}+R_{1} \\
N_{3} & =S_{3}+I_{3} \\
N_{2} & =S_{2}+E_{2}+I_{21}+I_{22}+R_{2}
\end{aligned}
$$

Table 1: Parameters of the Model

\begin{tabular}{ll}
\hline Parameter & Description \\
\hline$\Lambda_{1}$ & Number of recruitment into female population \\
$\omega_{1}$ & Proportion of births without microcephaly into female population \\
$\left(1-\omega_{1}\right)$ & Proportion of female births with microcephaly \\
$\theta_{1}$ & Proportion of susceptible female births without microcephaly \\
\hline
\end{tabular}




\begin{tabular}{|c|c|}
\hline$\left(1-\theta_{1}\right)$ & Proportion of exposed female births without Microcephaly \\
\hline$\mu_{1}$ & Natural death rate of females \\
\hline$\Lambda_{2}$ & Number of recruitment into male population \\
\hline$\omega_{2}$ & Proportion of births without microcephaly into male population \\
\hline$\left(1-\omega_{2}\right)$ & Proportion of male births with microcephaly \\
\hline$\theta_{2}$ & Proportion of susceptible female births without microcephaly \\
\hline$\left(1-\theta_{2}\right)$ & Proportion of exposed female births without Microcephaly \\
\hline$\mu_{2}$ & Natural death rate of males \\
\hline$\Lambda_{3}$ & Number of recruitment into the population of mosquitoes without zika virus \\
\hline$\mu_{3}$ & Natural death rate of mosquitoes \\
\hline$\delta$ & Death rate of mosquitoes due to insecticides \\
\hline$\alpha_{1}$ & Transmission rate of infection through mosquito bite to the susceptible females \\
\hline$\alpha_{2}$ & Transmission rate of infection through mosquito bite to the susceptible males \\
\hline$\alpha_{21}$ & Transmission rate of infection through sex from symptomatic infectious males to susceptible females \\
\hline$\alpha_{22}$ & Transmission rate of infection through sex from asymptomatic infectious males to susceptible females \\
\hline$\alpha_{11}$ & Transmission rate of infection through sex from symptomatic infectious females to susceptible males \\
\hline$\alpha_{12}$ & Transmission rate of infection through sex from symptomatic infectious females to susceptible males \\
\hline$\alpha_{3}^{11}$ & Transmission rate of virus from symptomatic infectious females to mosquitoes without virus through mosquito bite \\
\hline$\alpha_{3}^{12}$ & Transmission rate of virus from asymptomatic infectious females to mosquitoes without virus through mosquitoes bite \\
\hline$\alpha_{3}^{21}$ & Transmission rate of virus from symptomatic infectious males to mosquitoes without virus through mosquito bite \\
\hline$\alpha_{3}^{22}$ & Transmission rate of virus from asymptomatic infectious males to mosquitoes without virus through mosquito bite \\
\hline$\sigma_{11}$ & Progression rate of exposed females to the symptomatic infectious compartment \\
\hline$\sigma_{12}$ & Progression rate of exposed females to the asymptomatic infectious compartment \\
\hline$\sigma_{21}$ & Progression rate of exposed males to the symptomatic infectious compartment \\
\hline$\sigma_{22}$ & Progression rate of exposed males to the asymptomatic infectious compartment \\
\hline$\gamma_{1}$ & Rate of recovery from the compartment of exposed females to the removed compartment \\
\hline$\gamma_{2}$ & Rate of recovery from the compartment of exposed males to the removed compartment \\
\hline$\gamma_{11}$ & Rate of recovery from the compartment of symptomatic, infectious, females to the removed compartment \\
\hline$\gamma_{12}$ & Rate of recovery from the compartment of asymptomatic, infectious, females to the removed compartment \\
\hline$\gamma_{21}$ & Rate of recovery from the compartment of symptomatic, infectious, males to the removed compartment \\
\hline$\gamma_{22}$ & Rate of recovery from the compartment of asymptomatic, infectious, males to the removed compartment \\
\hline$\phi_{3}$ & $\begin{array}{l}\text { Is measuring the reduction in effectiveness of mosquito activities in transmitting virus by creating non conducive } \\
\text { environment for the mosquitoes through the use of air conditioner }\end{array}$ \\
\hline$\phi_{11}$ & Is measuring the reduction in effectiveness of sexual transmission through adherence to the preventive instructions \\
\hline$\phi_{12}$ & Is measuring the reduction in effectiveness of sexual transmission through adherence to the preventive instructions \\
\hline$\phi_{21}$ & Is measuring the reduction in effectiveness of sexual transmission through adherence to the preventive instructions \\
\hline$\phi_{22}$ & Is measuring the reduction in effectiveness of sexual transmission through adherence to the preventive instructions \\
\hline$\left(1-\in_{c} \tau_{c}\right)$ & $\begin{array}{l}\text { Reflects the impact of condom usage which is enhanced by public campaign (efficacy and compliance) on sexual } \\
\text { transmission where } 0<\epsilon_{c}, \tau_{c}<1\end{array}$ \\
\hline
\end{tabular}

\section{RESULTS AND DISCUSION}

Theorem 1: The Zika transmission model (1) - (12) has endemic equilibrium point if $R_{e}>1$.

Proof

$\varepsilon_{E}=\left(S_{1}^{* *}, E_{1}{ }^{* *}, I_{11}{ }^{* *}, I_{12}{ }^{* *}, R_{1}{ }^{* *}, S_{3}{ }^{* *}, I_{3}{ }^{* *}, S_{2}{ }^{* *}, E_{2}{ }^{* *}, I_{21}{ }^{* *}, I_{22}{ }^{* *}, R_{2}{ }^{* *}\right)$ is assumed to be the endemic point of (2) - (12) then the respective compartments are expressed in terms of parameters and forces of infection, 


$$
\begin{aligned}
& S_{1}^{* *}=\frac{\theta_{1} \omega_{1} \Lambda_{1}}{\eta \lambda_{H}^{* *}+\mu_{1}} \\
& E_{1}^{* *}=\frac{\omega_{1} \Lambda_{1} \eta \lambda_{H}^{* *}+\left(1-\theta_{1}\right) \omega_{1} \Lambda_{1} \mu_{1}}{k_{1}\left(\eta \lambda_{H}{ }^{* *}+\mu_{1}\right)} \\
& I_{11}^{* *}=\frac{\sigma_{11} \omega_{1} \Lambda_{1} \eta \lambda_{H}{ }^{* *}+\left(1-\theta_{1}\right) \sigma_{11} \omega_{1} \Lambda_{1} \mu_{1}}{k_{1} k_{2}\left(\eta \lambda_{H}{ }^{* *}+\mu_{1}\right)} \\
& I_{12}^{* *}=\frac{\sigma_{12} \omega_{1} \Lambda_{1} \eta \lambda_{H}^{* *}+\left(1-\theta_{1}\right) \sigma_{12} \omega_{1} \Lambda_{1} \mu_{1}}{k_{1} k_{3}\left(\eta \lambda_{H}{ }^{* *}+\mu_{1}\right)} \\
& R_{1}^{* *}=\frac{\left(k_{2} k_{3} \gamma_{1}+k_{3} \gamma_{11} \sigma_{11}+k_{2} \gamma_{12} \sigma_{12}\right)\left\{\omega_{1} \Lambda_{1} \eta \lambda_{H}{ }^{* *}+\left(1-\theta_{1}\right) \omega_{1} \Lambda_{1} \mu_{1}\right\}}{k_{1} k_{2} k_{3}\left(\eta \lambda_{H}{ }^{* *}+\mu_{1}\right)} \\
& S_{3}^{* *}=\frac{\Lambda_{3}}{\lambda_{3}^{* *}+k_{4}} \\
& I_{3}^{* *}=\frac{\Lambda_{3} \lambda_{3}^{* *}}{k_{4}\left(\lambda_{3}^{* *}+k_{4}\right)} \\
& S_{2}^{* *}=\frac{\theta_{2} \omega_{2} \Lambda_{2}}{(1-\eta) \lambda_{H}^{* *}+\mu_{2}} \\
& E_{2}^{* *}=\frac{\omega_{2} \Lambda_{2}(1-\eta) \lambda_{H}^{* *}+\left(1-\theta_{2}\right) \omega_{2} \Lambda_{2} \mu_{2}}{k_{5}\left[(1-\eta) \lambda_{H}{ }^{* *}+\mu_{2}\right]} \\
& I_{21}^{* *}=\frac{\sigma_{21} \omega_{2} \Lambda_{2}(1-\eta) \lambda_{H}^{* *}+\left(1-\theta_{2}\right) \sigma_{21} \omega_{2} \Lambda_{2} \mu_{2}}{k_{5} k_{6}\left[(1-\eta) \lambda_{H}{ }^{* *}+\mu_{2}\right]} \\
& I_{22}{ }^{* *}=\frac{\sigma_{22} \omega_{2} \Lambda_{2}(1-\eta) \lambda_{H}^{* *}+\left(1-\theta_{2}\right) \sigma_{22} \omega_{2} \Lambda_{2} \mu_{2}}{k_{5} k_{7}\left[(1-\eta) \lambda_{H}{ }^{* *}+\mu_{2}\right]} \\
& R_{2}^{* *}=\frac{\left(k_{6} k_{7} \gamma_{2}+k_{7} \gamma_{21} \sigma_{21}+k_{6} \gamma_{22} \sigma_{22}\right)\left\{\omega_{2} \Lambda_{2}(1-\eta) \lambda_{H}^{* *}+\left(1-\theta_{2}\right) \omega_{2} \Lambda_{2} \mu_{2}\right\}}{k_{5} k_{6} k_{7}\left[(1-\eta) \lambda_{H}^{* *}+\mu_{2}\right]} \\
& \lambda_{1}^{* *}=\eta \lambda_{H}^{* *} \\
& \lambda_{2}^{* *}=(1-\eta) \lambda_{H}{ }^{* *} \\
& \lambda_{H}^{* *}=\frac{\beta_{8} I_{3}{ }^{* *}+\beta_{2} I_{21}{ }^{* *}+\beta_{3} I_{22}{ }^{* *}+\beta_{9} I_{11}{ }^{* *}+\beta_{10} I_{12}{ }^{* *}}{N_{H}{ }^{* *}} \\
& \lambda_{3}^{* *}=\frac{\beta_{4} I_{11}{ }^{* *}+\beta_{5} I_{12}{ }^{* *}+\beta_{6} I_{21}{ }^{* *}+\beta_{7} I_{22}{ }^{* *}}{N_{3}^{* *}}
\end{aligned}
$$

$\lambda_{H}$ represents the force of infection from mosquitoes and infectious humans to susceptible humans. $\lambda_{3}^{* *}$ is the force of transmitting virus from infected humans to the vectors.

Substitute for $I_{11}{ }^{* *}, I_{12}{ }^{* *}, I_{21}{ }^{* *}, I_{22}{ }^{* *}$ and $I_{3}{ }^{* *}$ in (30) and (31) and simplify, then with $a_{i}{ }^{\prime} s$ definitions, for $i=1,2, \ldots 14$, equations (30) and (31) become 


$$
\left.\begin{array}{rl}
\lambda_{3}^{* *}= & \frac{a_{1} \lambda_{H}^{* *^{3}}+a_{2} \lambda_{H}^{* *^{2}}+a_{3} \lambda_{H}^{* *}+a_{4}}{a_{5} \lambda_{H}^{* *^{3}}+a_{6} \lambda_{H}^{* *^{2}}+a_{7} \lambda_{H}^{* *}+a_{8}} \\
\lambda_{3}^{* * *}= & \frac{a_{9} \lambda_{H}^{* *^{2}}+a_{10} \lambda_{H}^{* *}+a_{11}}{a_{12} \lambda_{H}^{* *^{2}}+a_{13} \lambda_{H}^{* *}+a_{14}} \\
a_{1}= & k_{1} k_{2} k_{3} k_{4}^{2} k_{5} k_{6} k_{7} N_{H}^{* *}(1-\eta) \\
& \left\{k_{1} k_{2} k_{3} k_{4}^{2} k_{5} k_{6} k_{7} N_{H}^{* *}\left[(1-\eta) \mu_{1}+\eta \mu_{2}\right]-k_{1} k_{2} k_{3} k_{4}^{2} \omega_{2} \Lambda_{2}(1-\eta) \eta\left(k_{7} \beta_{2} \sigma_{21}+k_{6} \beta_{3} \sigma_{22}\right)-\right]
\end{array}\right\}
$$

Equating equations (30) and (31) and simplifying the result gives

$$
\begin{aligned}
& \left.\begin{array}{c}
\left\{\left(a_{1} a_{12}-a_{5} a_{9}\right) \lambda_{H}^{* *^{5}}+\left[\left(a_{1} a_{13}+a_{2} a_{12}\right)-\left(a_{5} a_{10}+a_{6} a_{9}\right)\right] \lambda_{H}^{* *^{*}}+\left[\left(a_{1} a_{14}+a_{2} a_{13}+a_{3} a_{12}\right)-\left(a_{5} a_{11}+a_{6} a_{10}+a_{7} a_{9}\right)\right] \lambda_{H}^{* *^{3}}+\right. \\
\left.\left[\left(a_{2} a_{14}+a_{3} a_{13}+a_{4} a_{12}\right)-\left(a_{6} a_{11}+a_{7} a_{10}+a_{8} a_{9}\right)\right] \lambda_{H}^{* *^{2}}+\left[\left(a_{3} a_{14}+a_{4} a_{13}\right)-\left(a_{7} a_{11}+a_{8} a_{10}\right)\right] \lambda_{H}^{* *}+\left(a_{4} a_{14}-a_{8} a_{11}\right)\right\}
\end{array}\right]=0 \\
& \Rightarrow b_{5} \lambda_{H}^{* *^{5}}+b_{4} \lambda_{H}^{* *^{4}}+b_{3} \lambda_{H}^{* *^{3}}+b_{2} \lambda_{H}^{* *^{2}}+b_{1} \lambda_{H}^{* *}+b_{0}=0 \\
& \Rightarrow b_{5}>0, b_{4}>0, b_{3}>0, b_{2}>0 \text { and } b_{1}>0 \text {. } \\
& \Rightarrow \quad b_{0}=a-b<c\left(1-R_{e}\right)-b<0 \text { if and only if } R_{e}>1 \\
& \Rightarrow b_{5} \lambda_{H}^{* *^{5}}+b_{4} \lambda_{H}^{* *^{4}}+b_{3} \lambda_{H}^{* *^{3}}+b_{2} \lambda_{H}^{* *^{2}}+b_{1} \lambda_{H}^{* *}+\left[c\left(1-R_{e}\right)-b\right]=0 \text {, }
\end{aligned}
$$

\section{QED}

Conclusion: Invoking Descartes rule of signs to polynomial, states that if given a polynomial with real coefficients then the number of positive roots or zeros of the polynomial is equal the number of variation in sign of the polynomial (number of sign changes) or less than this by an even number. Applying the above 
rule of signs to the polynomial (47) shows that there is only one sign change. Hence the system (1) - (12) has a unique endemic equilibrium point, which is a confirmation of the existence of endemic state of the system (1) - (12).

\section{REFERENCES}

Agusto, FB; Bewick, S; Fagan, WF (2017). Mathematical model for Zika virus dynamics with sexual transmission route. Ecological complexity $29,61-81$.

Center for Disease Control and Prevention (CDC, 2016). Zika virus lives longer in semen than blood

European Centre for Disease Prevention and Control (ECDC, 2016). Factsheet for Health Professionals.

Funk, S; Kucharski, AJ; Camacho, A; Eggo, LY; Edmunds, WJ (2016). Comparative analysis of Dengue and Zika outbreaks reveals the differences by setting the virus Doi: http//dx.doi.org/10.1101/043265.

Gao, D; Lou, Y; He, D; Porce, TC; Kuang, Y; Chowell, G; Ruan, S (2016). Prevention and control of Zika a mosquito- borne and sexually transmitted disease. A Mathematical analysis. Scientific Reports. Nature Publishing Group 6. Doi: 10.1038/srep28070.

Kucharski, AJ; Funk, S; Eggo, RM; Mallet, H; Edmunds, WJ; Nilles, EJ (2016). Transmission Dynamics of Zika virus in Island populations: A modeling analysis of the 2013-2014 French Polynesia outbreaks. Eurosurveillance, 19, 20761.

World Health Organization (WHO, 2016) Emergency Committee on zika virus and observed increase in neurological disorders and neonatal malformations, Geneva, http://www.who.int/mediacentre/news/statement s/2016/zika -third -ec/en/. 